\title{
APPLYING CEGAR TO THE PETRI NET STATE EQUATION
}

\author{
HARRO WIMMEL AND KARSTEN WOLF
}

Universität Rostock, Institut für Informatik

e-mail address: \{harro.wimmel, karsten.wolf\}@uni-rostock.de

\begin{abstract}
We propose a reachability verification technique that combines the Petri net state equation (a linear algebraic overapproximation of the set of reachable states) with the concept of counterexample guided abstraction refinement. In essence, we replace the search through the set of reachable states by a search through the space of solutions of the state equation. We demonstrate the excellent performance of the technique on several real-world examples. The technique is particularly useful in those cases where the reachability query yields a negative result: While state space based techniques need to fully expand the state space in this case, our technique often terminates promptly. In addition, we can derive some diagnostic information in case of unreachability while state space methods can only provide witness paths in the case of reachability.
\end{abstract}

\section{INTRODUCTION}

Reachability is the fundamental verification problem. For place/transition Petri nets (which may have infinitely many states), it is one of the hardest decision problems known among the naturally emerging yet decidable problems in computer science. General solutions have been found by Mayr [14] and Kosaraju [9] with later simplifications made by Lambert [11], but there are complexity issues. All these approaches use coverability graphs which can have a non-primitive-recursive size with respect to the corresponding Petri net. A new approach by Leroux [12] not using such graphs gives some hope, but a concrete upper bound for the worst case complexity so far eludes us. In a sense even worse, Lipton [13] has shown that the problem is EXPSPACE-hard, so any try at programming a tool efficiently solving this problem to the full extent must surely fail.

Nevertheless, efficient tools exist that are applicable to a considerable number of problem instances. Model checkers, symbolic [3] or with partial order reduction [20, have been used successfully to solve quite large reachability problems. On a positive answer, a model checker can typically generate a trace, i.e. a firing sequence leading to the final marking. In contrast, negative answers are usually not accompanied by any diagnostic information. Such information, i.e. a counterexample or reasoning why the problem has a negative solution would require a deep analysis of the structure of the Petri net. So far, no tools are known that analyze the structure of a net and allow for such reasoning.

1998 ACM Subject Classification: F.2.2, I.6.4.

Key words and phrases: Petri Net, Reachability Problem, Integer Programming, CEGAR, Structure Analysis, Partial Order Reduction. 
This paper presents an approach to the reachability problem that combines two existing methods. First, we employ the state equation for Petri nets. This is a linear-algebraic overapproximation on the set of reachable states. Second, we use the concept of counterexample guided abstraction refinement (CEGAR) [4] for enhancing the expressiveness of the state equation. In essence, we iteratively analyse spurious solutions of the state equation and add constraints that exclude a solution found to be spurious but do not exclude any real solution. The approach has several advantages compared to (explicit or symbolic) purely state space based verification techniques:

- The search is quite focussed from the beginning as we traverse the solution space of the state equation rather than the set of reachable states;

- The search is close to breadth-first traversal, so small witness traces are generated;

- The method may perform well on unreachable problem instances (where state space techniques compute maximum size state spaces);

- In several unreachable problem instances, some kind of diagnostic information can be provided;

- A considerable workload can be shifted to very mature tools for solving linear programming problems.

In Sect. 2 we give the basic definitions. Section 3 shows how to use integer programming tools to find candidates for a solution. Section 4 deals with the analysis of the Petri net structure that is needed to push the integer programming onto the right path. In Sect. 5 we use methods of partial order reduction to mold the results of the integer programming into firing sequences solving the reachability problem. In Sect. 6 the overall algorithm is presented and in Sect. 7 we drop a few hints on how and when diagnostic information for unreachability can be generated. Finally, Sect. 8 compares the results of an implementation with another model checker, showing that structure analysis can compete with other approaches.

\section{The Reachability Problem}

Definition 2.1 (Petri net, marking, firing sequence). A Petri net $N$ is a tuple $(S, T, F)$ with a set $S$ of places, a set $T$ of transitions, where $S \neq \emptyset \neq T$ and $S \cap T=\emptyset$, and a mapping $F:(S \times T) \cup(T \times S) \rightarrow \mathbb{N}$ defining arcs between places and transitions.

A marking or state of a Petri net is a map $m: S \rightarrow \mathbb{N}$. A place $s$ is said to contain $k$ tokens under $m$ if $m(s)=k$. A transition $t \in T$ is enabled under $m, m[t\rangle$, if $m(s) \geq F(s, t)$ for every $s \in S$. A transition $t$ fires under $m$ and leads to $m^{\prime}, m[t\rangle m^{\prime}$, if additionally $m^{\prime}(s)=m(s)-F(s, t)+F(t, s)$ for every $s \in S$.

A word $\sigma \in T^{*}$ is a firing sequence under $m$ and leads to $m^{\prime}, m[\sigma\rangle m^{\prime}$, if either $m=m^{\prime}$ and $\sigma=\varepsilon$, the empty word, or $\sigma=w t, w \in T^{*}, t \in T$ and $\exists m^{\prime \prime}: m[w\rangle m^{\prime \prime}[t\rangle m^{\prime}$. A firing sequence $\sigma$ under $m$ is enabled under $m$, i.e. $m[\sigma\rangle$. The Parikh image of a word $\sigma \in T^{*}$ is the vector $\wp(\sigma): T \rightarrow \mathbb{N}$ with $\wp(\sigma)(t)=\#_{t}(\sigma)$, where $\#_{t}(\sigma)$ is the number of occurrences of $t$ in $\sigma$. For any firing sequence $\sigma$, we call $\wp(\sigma)$ realizable.

As usual, places are drawn as circles (with tokens as black dots inside them), transitions as rectangles, and arcs as arrows with $F(x, y)>0$ yielding an arrow pointing from $x$ to $y$. If an arc has a weight of more than one, i.e. $F(x, y)>1$, the number $F(x, y)$ is written next to the arc. In case $F(x, y)=F(y, x)>0$, we may sometimes draw a line with arrowheads at both ends. 
Note, that the Parikh image is not an injective function. Therefore, $\wp(\sigma)$ can be realizable even if $\sigma$ is not a firing sequence (provided there is another firing sequence $\sigma^{\prime}$ with $\left.\wp(\sigma)=\wp\left(\sigma^{\prime}\right)\right)$.

Definition 2.2 (Reachability problem). A marking $m^{\prime}$ is reachable from a marking $m$ in a net $N=(S, T, F)$ if there is a firing sequence $\sigma \in T^{*}$ with $m[\sigma\rangle m^{\prime}$. A tuple $\left(N, m, m^{\prime}\right)$ of a net and two markings is called a reachability problem and has the answer "yes" if and only if $m^{\prime}$ is reachable from $m$ in $N$. The set RP $=\left\{\left(N, m, m^{\prime}\right) \mid N\right.$ is a Petri net, $m^{\prime}$ is reachable from $m$ in $N\}$ is generally called the reachability problem, for which membership is to be decided.

It is well-known that a necessary condition for a positive answer to the reachability problem is the feasibility of the state equation.

Definition 2.3 (State equation). For a Petri net $N=(S, T, F)$ let $C \in \mathbb{N}^{S \times T}$, defined by $C_{s, t}=F(t, s)-F(s, t)$, be the incidence matrix of $N$. For two markings $m$ and $m^{\prime}$, the system of linear equations $m+C x=m^{\prime}$ is the state equation of $N$ for $m$ and $m^{\prime}$. A vector $x \in \mathbb{N}^{T}$ fulfilling the equation is called a solution.

Proposition 2.4. For any firing sequence $\sigma$ of a net $N=(S, T, F)$ leading from $m$ to $m^{\prime}$, i.e. $m[\sigma\rangle m^{\prime}$, holds $m+C \wp(\sigma)=m^{\prime}$, i.e. the Parikh vector of $\sigma$ is a solution of the state equation for $N, m$, and $m^{\prime}$.

This is just a reformulation of the firing condition for $\sigma$.

Proposition 2.5. If the Petri net is acyclic, i.e. the transitive closure of $F$ is irreflexive then the existence of a solution of the state equation for $N, m$, and $m^{\prime}$ is a sufficient condition for reachability of $m^{\prime}$ from $m$ in $N$.

In an acyclic net, minimal transitions (with respect to $F$ ) that occur in the support of a solution $x$ of the state equation must be enabled under $m$. Firing such a transition with resulting marking $m^{*}$ leads to a smaller reachability problem $\left(N, m^{*}, m^{\prime}\right)$ that has $x$ minus one occurrence of $t$ as a solution. By induction, the whole solution can be unwound to a firing sequence.

In Petri nets with cycles, it is possible to have a sequence $\sigma$ such that its Parikh image fulfills the state equation but it is not a firing sequence. The easiest example for this occurs in a net $N=(\{s\},\{t\}, F)$ with $F(s, t)=1=F(t, s)$. Let $m$ and $m^{\prime}$ be the empty marking, i.e. one with zero tokens overall, then $m[t\rangle m^{\prime}$ is obviously wrong but $m+C \wp(\sigma)=m^{\prime}$ holds since $C=(0)$. The effect can occur whenever the Petri net contains a cycle of transitions. Interestingly, certain cycles of transitions can also help to overcome this problem, see Fig. 1. Here, we would like to fire a word $t t^{\prime}$ from the marking $m$ with $m\left(s_{1}\right)=m\left(s_{2}\right)=0$ and $m\left(s_{3}\right)=1$, but obviously, this is impossible. If we interleave, however, $t t^{\prime}$ with the sequence $u u^{\prime}$ we can fire $u t t^{\prime} u^{\prime}$. The sequence $u t t^{\prime} u^{\prime}$ corresponds to a solution of the state equation for $N, m$, and $m^{\prime}$ that is not minimal. More precisely, we have $C \wp\left(u u^{\prime}\right)=0$. At first glance, such a sequence does not change the marking and appears to be neglegible. However, it has a valuable effect on $t t^{\prime}$ in "lending a token" to $s_{2}$. The transition $u$ provides that token, $u^{\prime}$ takes it back, but meanwhile the token helps the sequence $t t^{\prime}$ to proceed. The process of "lending tokens" is not visible in the state equation as the latter overapproximates the token game of Petri nets to linear algebra. In consequence, it is necessary for our approach to consider non-minimal solutions of the state equation and in particular solutions to the corresponding homogeneous system of equations. 


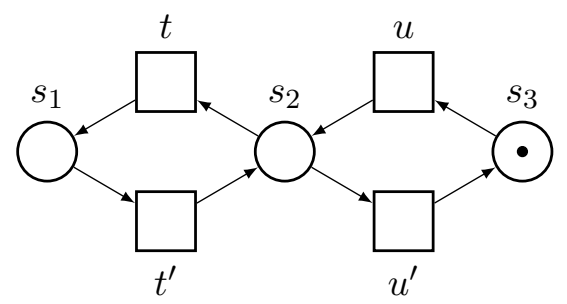

$$
\left(\begin{array}{rrrr}
t & t^{\prime} & u & u^{\prime} \\
1 & -1 & 0 & 0 \\
-1 & 1 & 1 & -1 \\
0 & 0 & -1 & 1
\end{array}\right) s_{1}
$$

Figure 1: The word $t t^{\prime}$ cannot fire, but we can borrow a token from the circle $u u^{\prime}$, so $u t t^{\prime} u^{\prime}$ can fire and leads to the same marking as $t t^{\prime}$. The incidence matrix of the net is shown on the right

Definition 2.6 (T-invariant). Let $N=(S, T, F)$ be a Petri net and $C$ its incidence matrix. A vector $x \in \mathbb{N}^{T}$ is called a $T$-invariant if $C x=0$. If a T-invariant corresponds to some executable firing sequence, it is called realizable.

A realizable $T$-invariant represents a cycle in the state space. Corresponding firing sequences do not change the marking. However, its interleaving with another sequence $\sigma$ may turn $\sigma$ from unrealizable to realizable.

Solving the state equation is a non-negative integer programming problem. From linear algebra we know that the solution space is semi-linear.

Corollary 2.7 (Solution space). For a given state equation $m+C x=m^{\prime}$ over a net $N=(S, T, F)$, there are numbers $j, k \in \mathbb{N}$ and finite sets of vectors $B=\left\{b_{i} \in \mathbb{N}^{T} \mid 1 \leq i \leq j\right\}$ (base vectors) and $P=\left\{p_{i} \in \mathbb{N}^{T} \mid 1 \leq i \leq k\right\}$ (period vectors) such that:

- all $b_{i} \in B$ are pairwise incomparable (by standard componentwise comparison for vectors) and thus minimal solutions,

- $P$ forms a basis for the non-negative solution space $P^{*}=\left\{\sum_{i=1}^{k} n_{i} p_{i} \mid n_{i} \in \mathbb{N}, p_{i} \in P\right\}$ of $C x=0$,

- for all solutions $x$ there are $n_{i} \in \mathbb{N}$ for $1 \leq i \leq k$ and $n \in\{1, \ldots, j\}$ such that $x=$ $b_{n}+\sum_{i=1}^{k} n_{i} p_{i}$

- for every solution $x$, all vectors of the set $x+P^{*}$ are solutions as well.

Note that only linear combinations with nonnegative coefficients are considered in this representation. In this setting, the number of base vectors as well as the number of period vectors may exponentially depend on the size of the net. Permitting negative combinations (and thus solutions in the integers instead of the natural numbers) would yield a significant loss in precision of the state equation.

So we know that all solutions can be obtained by taking a minimal solution $b$ of the state equation and adding a linear combination of $T$-invariants from some basis $P$. Usually, not all the elements from $B$ and $P$ we use for a solution are realizable, though. While the sum of two realizable $T$-invariants remains realizable (just concatenate the according firing sequences as they have identical initial and final marking), the sum of two non-realizable $T$-invariants may well become realizable. This can be seen in Fig. 2, where neither $\wp\left(t t^{\prime}\right)$ nor $\wp\left(u u^{\prime}\right)$ is realizable under the marking $m$ with $m\left(s_{1}\right)=m\left(s_{4}\right)=1$ and $m\left(s_{2}\right)=m\left(s_{3}\right)=0$, but the sequence $t u t^{\prime} u^{\prime}$ realizes $\wp\left(t t^{\prime} u u^{\prime}\right)$. The matter is even more complicated when a minimal solution from $B$ is introduced, because positive minimal solutions are never $T$ invariants (unless $m=m^{\prime}$ ), i.e. they change the marking of the net, so their realizations cannot just be concatenated. 


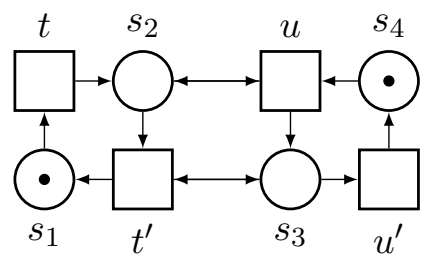

Figure 2: Neither the T-invariant $\wp\left(t t^{\prime}\right)$ nor $\wp\left(u u^{\prime}\right)$ is realizable, but $\wp\left(t t^{\prime} u u^{\prime}\right)$ is, by the sequence $t u t^{\prime} u^{\prime}$

\section{Traversing the Solution Space}

Though realizability of transition vectors is and remains a problem, the first real problem we encounter when we try to solve the state equation is a practical one. While there are solvers that can determine the complete sets of base and period vectors for the solution space, e.g. 4 ti2 [1], these programs can only do that for very small systems. If the system has a hundred or more variables, we are out of luck. On the other hand, integer programming (IP) solvers like $l p \_s o l v e$ [2] are much faster but will only find one solution to the state equation at a time.

Another point of interest to know about IP solvers is the objective function, which can be any function over the variables of the linear system to solve. While looking for a solution, the solver tries to minimize or maximize this function. Minimizing seems more valuable here, since we might use the sum over all variables as our objective function, effectively telling the solver to produce a solution that would lead to a shortest firing sequence if realizable. In the following, we will assume such an objective function.

Fortunately, we can force an IP solver to produce more than just one solution - this is the CEGAR part of our approach. If a solution found is not realizable, we may add an inequation to our state equation to forbid that solution. Starting the IP solver again will then lead to a different solution. The trick is, of course, to add inequations in such a way that no realizable solution is lost.

Definition 3.1 (Constraints). Let $N=(S, T, F)$ be a Petri net. We define two forms of constraints, both being linear inequations over transitions:

- a jump constraint takes the form $t<n$ with $n \in \mathbb{N}$ and $t \in T$. In general, it is intended to switch (jump) to another base solution, exploiting the incomparability of different minimal base solutions.

- an increment constraint takes the form $\sum_{i=1}^{k} n_{i} t_{i} \geq n$ with $n_{i} \in \mathbb{Z}, n \in \mathbb{N}$, and $t_{i} \in T$. Among others, it can be used to force non-minimal solutions.

To understand the idea for differentiating between these two forms of constraints, it is necessary to introduce the concept of a partial solution first. A partial solution is obtained from a solution of the state equation under given constraints by firing as many transitions as possible.

Definition 3.2 (Partial solution). Let $N=(S, T, F)$ be a Petri net and $\Omega$ a total order over $\mathbb{N}^{T}$ that includes the partial order given by $x<y$ if $\sum_{t \in T} x(t)<\sum_{t \in T} y(t)$. A partial solution of a reachability problem $\left(N, m, m^{\prime}\right)$ is a tuple $(\Gamma, x, \sigma, r)$ of

- a family of (jump and increment) constraints $\Gamma=\left(c_{1}, \ldots, c_{n}\right)$, 
- the $\Omega$-smallest solution $x$ fulfilling the state equation of $\left(N, m, m^{\prime}\right)$ and the constraints of $\Gamma$,

- a firing sequence $\sigma \in T^{*}$ with $m[\sigma\rangle$ and $\wp(\sigma) \leq x$,

- a remainder $r$ with $r=x-\wp(\sigma)$ and $\forall t \in T:(r(t)>0 \Longrightarrow \neg m[\sigma t\rangle)$.

The vectors $x$ and $r$ are included for convenience only, they can be computed from $\Gamma, \sigma, \Omega$, and the problem instance.

A full solution is a partial solution $(\Gamma, x, \sigma, r)$ with $r=0$. In this case, $\sigma$ is a firing sequence solving our reachability problem (with answer 'yes').

We choose $\Omega$ such that an IP solver can be assumed to always produce the $\Omega$-smallest solution that does not contradict its linear system of equations. Note that from any firing sequence solving our reachability problem we can easily deduce a full solution:

Corollary 3.3 (Realizable solutions are full solutions). For any realizable solution $x$ of the state equation (realized by a firing sequence $\sigma$ ) we find a full solution $(\Gamma, x, \sigma, 0)$ where $\Gamma$ consists of constraints $t \geq x(t)$ for every $t$ with $x(t)>0$, and $\wp(\sigma)=x$.

Note, that $x$ is the smallest solution fulfilling $\Gamma$ and therefore also the $\Omega$-smallest solution.

By adding a constraint to a partial solution we may obtain new partial solutions (or not, if the linear system becomes infeasible). Any full solution can eventually be reached by consecutively extending an $\Omega$-minimal partial solution with constraints. The following lemma is thus a core argument for the correctness of our approach.

Lemma 3.4 (A path to a full solution). Let $b$ be the $\Omega$-minimal solution of the state equation of a reachability problem $\left(N, m, m^{\prime}\right)$ and $p s^{\prime}=\left(\left(c_{j}\right)_{1 \leq j \leq \ell}, b^{\prime}+\sum_{i=1}^{k} n_{i} p_{i}, \sigma^{\prime}, 0\right)$ a full solution of the problem. For $0 \leq n \leq \ell$, there are partial solutions $p s_{n}=\left(\left(c_{j}\right)_{1 \leq j \leq n}, x_{n}, \sigma_{n}, r_{n}\right)$ with $p s_{0}=\left(\emptyset, b, \sigma_{0}, r_{0}\right), p s_{\ell}=p s^{\prime}$, and $x_{n_{1}} \leq_{\Omega} x_{n_{2}}$ for $n_{1} \leq n_{2}$.

Proof. Let $\Gamma_{n}=\left(c_{j}\right)_{1 \leq j \leq n}$. If $p s_{n_{1}}, p s_{n_{2}}$ are two partial solutions (with $1 \leq n_{1}<n_{2} \leq \ell$ ) then $x_{n_{2}}$ is a solution of the state equation plus $\Gamma_{n_{1}}$, since it even fulfills the state equation plus $\Gamma_{n_{2}}$ with $\Gamma_{n_{1}} \subseteq \Gamma_{n_{2}}$. As $x_{n_{1}}$ is the $\Omega$-smallest solution of the state equation plus $\Gamma_{n_{1}}$, $x_{n_{1}} \leq_{\Omega} x_{n_{2}}$ holds. Therefore, $b \leq_{\Omega} x_{1} \leq_{\Omega} \ldots \leq_{\Omega} x_{\ell}$. Since $x_{\ell}=b^{\prime}+\sum_{i=1}^{k} n_{i} p_{i}$ is an existing solution of the strictest system, i.e. state equation plus $\Gamma_{\ell}$, each system of state equation plus one family of constraints $\Gamma_{n}$ is solvable. As a $\sigma_{n}$ can be determined by just firing transitions as long as possible, all the partial solutions $p s_{n}$ exist.

Now, let us assume a partial solution $p s=(\Gamma, x, \sigma, r)$ that is not a full solution, i.e. $r \neq 0$. Obviously, some transitions cannot fire often enough. There are three possible remedies for this situation:

(1) $x$ may still be realizable by a different firing sequence that corresponds to $x$. That is, we can find a full solution $p s^{\prime}=\left(\Gamma, x, \sigma^{\prime}, 0\right)$ with $\wp\left(\sigma^{\prime}\right)=x$.

(2) We can add a jump constraint to obtain an $\Omega$-greater solution vector for a different partial solution.

(3) If $r(t)>0$ for some transition $t$, we can add an increment constraint to increase the maximal number of tokens available on a place in the preset of $t$. Since the final marking remains the same, this means to borrow tokens for such a place. This can be done by adding a $T$-invariant containing the place to the solution.

A visualization of these ideas can be seen in Fig. 3 where $b$ denotes the $\Omega$-smallest solution. The cone over $b$ represents all solutions $b+P^{*}$ with $P$ being the set of period vectors, i.e. 


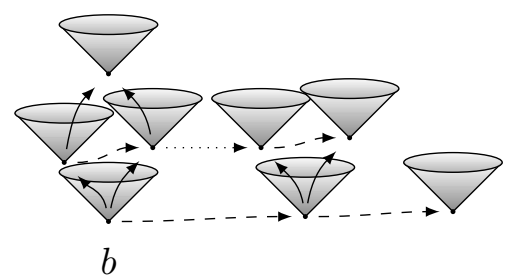

Figure 3: Paths from the $\Omega$-minimal solution $b$ to any solution. Black dots represent solutions, cones stand for linear solution spaces over such solutions, which may or may not intersect or include each other. Normal arrows increment a solution by adding a $T$-invariant, dashed arrows are jumps to an incomparable $\Omega$-greater solution. Such jumps can also occur on higher levels of linear solution spaces, shown by the dotted arrow

$T$-invariants. Jump constraints lead along the dashed or dotted lines to the next $\Omega$-minimal solution while normal arrows representing increment constraints lead upwards to show the addition of a $T$-invariant. How to build constraints doing just what we want them to do is the content of the next section.

\section{Building Constraints}

Let us first argue that for a state equation, any of the minimal solution vectors in $B$ can be obtained by using jump constraints.

Lemma 4.1 (Jumps to minimal solutions). Let $b, b^{\prime} \in B$ be base vectors of the solution space of the state equation $m+C x=m^{\prime}$ plus some set of constraints $\Gamma$. Assume $b$ to be the $\Omega$-minimal solution of the system. Then, we can obtain $b^{\prime}$ as output of our IP solver by consecutively adding jump constraints of the form $t_{i}<n_{i}$ with $n_{i} \in \mathbb{N}$ to $\Gamma$.

Proof. We know $b \leq_{\Omega} b^{\prime}$ holds, but since $b^{\prime}$ is a minimal solution, $b \leq b^{\prime}$ cannot hold. Therefore, a transition $t$ with $b^{\prime}(t)<b(t)$ must exist. After adding the constraint $t<b(t)$ to $\Gamma$ the IP solver can no longer generate $b$ as a solution. Assume $b^{\prime \prime}$ is the newly generated solution. If $b^{\prime}=b^{\prime \prime}$ we are done. Otherwise, since $b^{\prime}$ fulfills $t<b(t)$, it is still a solution of our system, and also a minimal one as the solution space is restricted by the added constraint. Thus, $b^{\prime \prime} \leq_{\Omega} b^{\prime}$ holds and we may recursively use the same argument as above for $b:=b^{\prime \prime}$. Since there are only finitely many solutions $\Omega$-smaller than $b^{\prime}$, the argument must terminate reaching $b^{\prime}$.

Non-minimal solutions may not be reachable this way, since the argument " $b^{\prime}(t)<b(t)$ for some $t$ " does not necessarily hold. We will need increment constraints for this, but unluckily, increment constraints and jump constraints may contradict each other. Assume our state equation has a solution of the form $b^{\prime}+p$ with a period vector $p \in P$ and to obtain $b^{\prime} \in B$ from the $\Omega$-minimal solution $b \in B$ we need to add (at least) a jump constraint $t_{i}<n_{i}$ to the state equation. If $p$ contains $t_{i}$ often enough, we will find that $\left(b^{\prime}+p\right)\left(t_{i}\right) \geq n_{i}$ holds. Therefore, $b^{\prime}+p$ is not a solution of the state equation plus the constraint $t_{i}<n_{i}$, i.e. adding an increment constraint demanding enough occurrences of $t_{i}$ for $b^{\prime}+p$ will render the linear equation system infeasible. The only way to avoid this problem is to remove the jump constraints before adding increment constraints. 
Lemma 4.2 (Transforming jumps). Let $z$ be the $\Omega$-minimal solution of the state equation $m+C x=m^{\prime}$ plus some constraints $\Gamma$. Let $\Gamma^{\prime}$ consist of all increment constraints of $\Gamma$ plus a constraint $t \geq z(t)$ for each transition $t$. Then, for all $y \geq z$, $y$ is a solution of $m+C x=m^{\prime}$ plus $\Gamma \cap \Gamma^{\prime}$ if and only if $y$ is a solution of $m+C x=m^{\prime}$ plus $\Gamma^{\prime}$. Furthermore, no $\Omega$-smaller solution of $m+C x=m^{\prime}$ plus $\Gamma$ than $z$ solves $m+C x=m^{\prime}$ plus $\Gamma^{\prime}$.

Proof. Let $y \geq z$ be a solution of $m+C x=m^{\prime}$ plus $\Gamma \cap \Gamma^{\prime}$. The additional constraints in $\Gamma^{\prime}$ only demand $y(t) \geq z(t)$, which is obviously the case. The other direction is trivial. For the second part, let $z^{\prime} \leq_{\Omega} z$ with $z \neq z^{\prime}$ be some solution of $m+C x=m^{\prime}$ plus $\Gamma$. Since $\sum_{t} z^{\prime}(t) \leq \sum_{t} z(t)$ (following from $\Omega$ ) but $z \neq z^{\prime}$, for at least one transition $t$ holds $z^{\prime}(t)<z(t)$. Due to the constraint $t \geq z(t)$ in $\Gamma^{\prime}, z^{\prime}$ cannot be a solution of $m+C x=m^{\prime}$ plus $\Gamma^{\prime}$.

As a consequence, if we are only interested in solutions of the cone $z+P^{*}$ over $z$, we can add increment constraints guaranteeing solutions greater or equal than $z$ and remove all jump constraints without any further restriction. Our IP solver will yield $z$ as the $\Omega$-minimal solution for both families of constraints, $\Gamma$ and $\Gamma^{\prime}$, and we can add further constraints leading us to any solution in the cone $z+P^{*}$ now.

Let $p s=(\Gamma, x, \sigma, r)$ now be a partial solution with $r>0$. We would like to determine sets of places that need additional tokens (and the number of these tokens) that would enable us to fire the remainder $r$ of transitions. Obviously, this problem is harder than the original problem of finding out if a transition vector is realizable, i.e. just testing if zero additional tokens are sufficient. A recursive approach would probably be very inefficient as for every solution $x$ there may be many different remainders $r$. Even though the remainders are smaller than the solution vector $x$, the number of recursion steps might easily grow exponentially with the size of $x$, i.e. $\sum_{t} x(t)$. We therefore adopt a different strategy, namely finding good heuristics to estimate the number of tokens needed. If a set of places actually needs $n$ additional tokens with $n>0$, our estimate may be any number from one to $n$. If we guess too low, we will obtain a new partial solution allowing us to make a guess once again, (more or less) slowly approaching the correct number. We propose a two-part algorithm. The first part computes sets of places and transitions that are of interest, the second estimates the number of tokens. For the first part, we use a dependency graph that is known from partial order reduction approaches [18. For every disabled transition, we can choose an insufficiently marked place. For every such place, we consider its pretransitions. Applying this idea to a partial solution (where all transitions in $r$ are disabled), this graph yields cycles, or more generally strongly connected components) of mutually blocked transitions and their insufficiently marked scapegoat places. In order to make parts of $r$ fireable, we need to interleave $r$ with a T-invariant that is able to lend tokens to any of the involved scapegoat places. Obviously, source SCC (i.e. SCC without incoming edges) are of particular interest for enabling parts of $r$. The following algorithm computes sets of places where additional tokens are necessary.

input: Reachability prob. $\left(N, m, m^{\prime}\right)$; partial solution $p s=(\Gamma, x, \sigma, r)$

output: A set of tuples $\left(S_{i}, T_{i}, X_{i}\right)$ with $S_{i} \subseteq S, T_{i} \cup X_{i} \subseteq T$

Determine $\hat{m}$ with $m[\sigma\rangle \hat{m}$;

Build a bipartite graph $G=\left(S_{0} \cup T_{0}, E\right)$ with

$T_{0}:=\{t \in T \mid r(t)>0\} ; S_{0}:=\left\{s \in S \mid \exists t \in T_{0}: \quad F(s, t)>\hat{m}(s)\right\} ;$

$E:=\left\{(s, t) \in S_{0} \times T_{0} \mid F(s, t)>\hat{m}(s)\right\} \cup\left\{(t, s) \in T_{0} \times S_{0} \mid F(t, s)>F(s, t)\right\} ;$

Calculate the strongly connected components (SCCs) of $G$; 


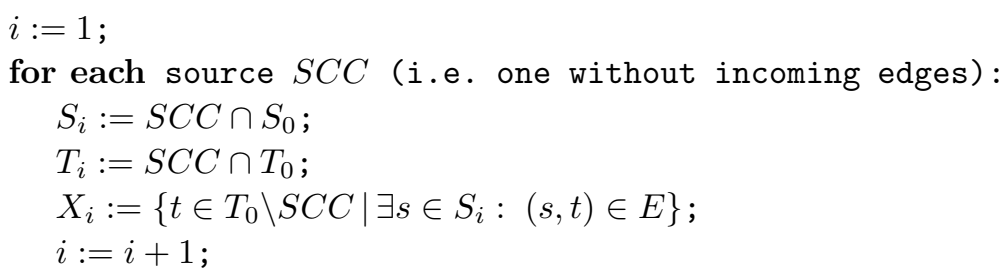

end for

The edges of the graph $G$ constructed in the algorithm have a different meaning depending on their direction. Edges from transitions to places signal that the transition would increase the number of tokens on the place upon firing, while edges in the other direction show the reason for the non-enabledness of the transition. A source SCC, i.e. a strongly connected component without incoming edges from other components, can therefore not obtain tokens by the firing of transitions from other SCCs. This means, tokens must come from somewhere else, that is, from firing transitions not appearing in the remainder $r$. For each set of places $S_{i}$ such identified as non-markable by the remainder itself, there are two sets of transitions. If one transition from the set $T_{i}$ would become firable, it is possible that all other transitions could fire as well, since the former transition effectively produces tokens on some place in the component. If the set $T_{i}$ is empty (the SCC consisting of a single place), the transitions mentioned in $r$ will not produce any tokens on the SCC. Thus, the token needs of the transitions depending on this SCC, i.e. those in $X_{i}$, must all be fulfilled together, since they cannot activate each other. Overall, we obtain:

Lemma 4.3. The previous algorithm determines source $S C C s\left(S_{i}, T_{i}\right)$ with insufficient numbers of tokens to enable the remainder $r$ of a partial solution as well as sets $X_{i}$ of transitions depending on those SCCs for firing.

Since enough additional tokens to enable one transition from a set $T_{i}$ might later enable the rest of $T_{i}$ and $X_{i}$ as well, it is difficult to compute the exact number of tokens needed on some SCC and where to place them. The following algorithm thus is a heuristic to determine a least number of tokens necessary on a source SCC.

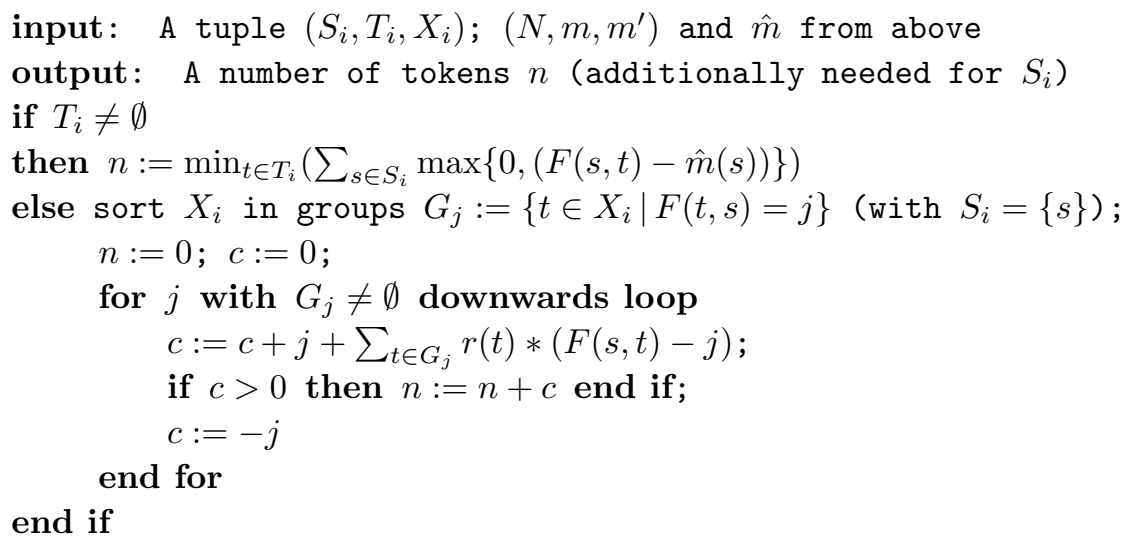

Lemma 4.4. For each set of places $S_{i}$ that need additional tokens according to the first part of the algorithm, the second part estimates that number of tokens (in a range from one to the actual minimum number of tokens necessary).

Proof. Consider a triple $\left(S_{i}, T_{i}, X_{i}\right)$ computed by the first algorithm. If $T_{i}$ is not empty, only line 4 of the second algorithm is executed, computes the number of tokens missing for each 
of the transitions in $T_{i}$, and takes the minimum over these numbers. By construction, this number is at least one and any lower number would not activate any transition from $T_{i}$.

If $T_{i}$ is empty, all transitions in $X_{i}$ depend on a single place which must provide all the necessary tokens to fire all the transitions in $X_{i}$. Note that while the transitions in $X_{i}$ all effectively consume tokens from $s \in S_{i}$, they may also put tokens back onto this place due to a loop. By firing those transitions with the lowest $F(t, s)$-values last, we minimize the leftover. Transitions with the same $F(t, s)$-value $j$ can be processed together, each consuming effectively $F(s, t)-j$ tokens except for the "first" transition which requires the existence of $j$ additional tokens. If some group $G_{j}$ of transitions leaves tokens on $s$, the next group can consume them, which is memorized in the variable $c$ (for carryover or consumption). Overall, we get the minimal number of tokens necessary to fire all transitions in $X_{i}$.

Observe, that the algorithm cannot return zero or negative values: There must be at least one transition in $T_{i} \cup X_{i}$, otherwise there would be no transition that cannot fire due to a place in $S_{i}$ and the places in $S_{i}$ would not have been computed at all. If $T_{i}$ is not empty, line 4 in the algorithm minimizes over positive values, i.e. the numbers of tokens missing for each transition in $T_{i}$; if $T_{i}$ is empty, line 8 will set $c$ to a positive value at its first execution, yielding a positive value for $n$.

We can thus try to construct a constraint from a set of places $S_{i}$ generated by the first part of the algorithm and the token number calculated in the second part. Since our state equation has transitions as variables, we must transform our condition on places into one on transitions first.

Lemma 4.5. Let $N=(S, T, F)$ be a Petri net, $\left(N, m, m^{\prime}\right)$ the reachability problem to be solved, $p s=(\Gamma, x, \sigma, r)$ a partial solution with $r>0$, and $\hat{m}$ the marking reached by $m[\sigma\rangle \hat{m}$. Let $S_{i}$ be a set of places and $n$ a number of tokens to be generated on $S_{i}$. Further, let $T_{i}:=\left\{t \in T \mid r(t)=0 \wedge \sum_{s \in S_{i}}(F(t, s)-F(s, t))>0\right\}$. We define a constraint $c$ by

$$
\sum_{t \in T_{i}} \sum_{s \in S_{i}}(F(t, s)-F(s, t)) t \geq n+\sum_{t \in T_{i}} \sum_{s \in S_{i}}(F(t, s)-F(s, t)) \wp(\sigma)(t) .
$$

Then, for the system $m+C x=m^{\prime}$ plus $\Gamma$ plus $c$, if our IP solver can generate a solution $x+y$ (y being a T-invariant) we can obtain a partial solution $\mathrm{ps}^{\prime}=(\Gamma \cup\{c\}, x+y, \sigma \tau, r+z)$ with $\wp(\tau)+z=y$. Furthermore, $\sum_{t \in T} \sum_{s \in S_{i}}(F(t, s)-F(s, t)) y(t) \geq n$.

Proof. (Sketch) First, note that $T_{i}$ contains the transitions that produce more on $S_{i}$ than they consume, but we have explicitly excluded all transitions of the remainder $r$, since we do not want the IP solver to increase the token production on $S_{i}$ by adding transitions that could not fire anyway. I.e., we would like to have a chance to fire the additional transitions in $y$ at some point, though there are no guarantees. The left hand side of $c$ contains one instance of a transition $t$ for each token that $t$ effectively adds to $S_{i}$. If we apply some transition vector $u$ to the left hand side of $c$ (i.e. replacing $t$ by $u(t)$ for each transition $t \in T_{i}$ ), we therefore get the number of tokens added to $S_{i}$ by firing the transitions from $T_{i}$ in $u$. Of course, other transitions in $u$ (outside $T_{i}$ ) might reduce this number again. For the right hand side of $c$, we calculate how many tokens are actually added to $S_{i}$ by the transitions from $T_{i}$ in the firing sequence $\sigma$ (and therefore also in the solution $x$ ) and increase that number by the $n$ extra tokens we would like to have. Since the extra tokens cannot come from $x$ in a solution $u:=x+y$, they must be produced by 
$y$, i.e. $\sum_{t \in T} \sum_{s \in S_{i}}(F(t, s)-F(s, t)) y(t) \geq n$. We might be able to fire some portion of $y$ after $\sigma$, resulting in the obvious $\wp(\tau)+z=y$.

When we apply our constraint we might get less or even more than the $n$ extra tokens, depending on the $T$-invariants in the net. There are three possible outcomes when we apply the new constraint: The constraint might be unsatisfiable, we might detect that the new constraint has not brought us any closer to a solution, or some of the remainder transitions can now fire (or have at least come closer to firing). In the first two cases, our partial solution just cannot be extended to a full solution, nothing is lost if we throw it away. Failing to detect the second case will not cut off any solutions but might prohibit termination. In the third case, we continue extending the partial solution with the help of further constraints. Therefore, if a solution exists, we can still find it, and we know that we can find it with the help of jump and increment constraints, since we only add necessary constraints. Further jump constraints may be necessary as there may be many incomparable, minimal solutions fulfilling the latest increment constraint.

Theorem 4.6 (Reachability of solutions). If a reachability problem has a solution, a realizable solution of the solution space of the state equation can be reached by consecutively adding constraints to the system of equations, always transforming jump constraints before adding increment constraints. This even holds, given some solution $x$, if we limit the increment constraints to those built by lemma 4.5 and jump constraints to those of the form $t<x(t)$.

Proof. (Sketch) The first sentence is obvious from lemma 3.4. For the second part, let $\sigma$ be a realizable solution with $\wp(\sigma)=x$ and let $b$ be a minimal solution with $b \leq x$. We can obtain $b$ by a set of jump constraints according to lemma 4.1. Either $b$ is realizable and we are done, or $b$ is not realizable. In the latter case, we compute an increment constraint forcing additional tokens to undermarked places according to lemma 4.3, 4.4, and 4.5, not losing any full solutions. Let $y$ be the solution computed for this extended system, then $b \leq y$ holds. If $y$ is realizable, we are done. If $y \leq x$, we add the next increment constraint. Otherwise, we test further jump constraints now and obtain all solutions $y_{1}, \ldots, y_{n}$ fulfilling the increment constraint but being incomparable to $y$. For one of them, $y_{k} \leq x$ must hold (as we have not lost any full solutions). We continue by checking realizability of $y_{k}$ and, in the negative case, repeat adding a necessary increment constraint. Since $x$ is finite and each constraint leads only to bigger solutions, at some point we must find a realizable solution or reach $x$.

\section{Finding Partial Solutions}

Producing partial solutions $p s=(\Gamma, x, \sigma, r)$ from a solution $x$ of the state equation (plus $\Gamma$ ) is actually quite easily done by brute force. We can build a tree with marking-annotated nodes and the firing of transitions as edges, allowing at most $x(t)$ instances of a transition $t$ on any path from the root of the tree to a leaf. Any leaf is a new partial solution from which we may generate new solutions by adding constraints to the state equation and forwarding the evolving linear system to our IP solver. If we just make a depth-first-search through our tree and backtrack at any leaf, we build up all possible firing sequences realizable from $x$. This is obviously possible without explicitly building the whole tree at once, thus saving memory. Of course, the tree might grow exponentially in the size of the solution vector $x$ 
and so some optimizations are in order to reduce the run-time. We would like to suggest a few ones here, especially partial order reductions.

(1) The stubborn set method [10] determines a set of transitions that can be fired before all others by investigating conflicts and dependencies between transitions at the active marking. The stubborn set is often much smaller than the set of enabled transitions under the same marking, leading to a tree with a lower degree. In our case, in particular the version of [15] is useful as, using this method, the reduced state space contains, for each trace to the target marking, at least one permution of the same trace. Hence, the reduction is consistent with the given solution of the state equation.

(2) Especially if transitions should fire multiple times $(x(t)>1)$ we observe that the stubborn set method alone is not efficient. The situation in Fig. 4 may occur quite often. Assume we reach some marking $\hat{m}$ by a firing sequence $\alpha$, so that transitions $t$ and $u$ are enabled. After proceeding through the subtree behind $t$ we backtrack to the same point and now fire $u$ followed by some sequence $\sigma$ after which $t$ is enabled, leading to $m[\alpha\rangle \hat{m}[u \sigma t\rangle \widetilde{m}$. If $\hat{m}[t \sigma u\rangle$ holds, we know that it reaches the same marking $\tilde{m}$ and the same remainder $r$ of transitions still has to fire. Therefore, in both cases the future is identical. Since we have already investigated what happens after firing $\alpha t \sigma u$, we may backtrack now omitting the subtree after $\alpha u \sigma t$. Note that a test if $\hat{m}[t \sigma u\rangle$ holds is quite cheap, as only those places $s$ with $C_{s, t}<C_{s, u}$ can prevent the sequence $t \sigma$. Enabledness of $u$ after $t \sigma$ can be tested by reverse calculating $\widetilde{m}_{1}=\widetilde{m}-C u$ and checking whether $\widetilde{m}_{1}$ is a marking and $\widetilde{m}_{1}[u\rangle \widetilde{m}$ holds.

(3) There are situations where a leaf belongs to a partial solution $p s^{\prime}$ that cannot lead to a (new) full solution. In this case the partial solution does not need to be processed. If we already tried to realize $x$ yielding a partial solution $p s=(\Gamma, x, \sigma, r)$ and $p s^{\prime}=$ $(\Gamma \cup\{c\}, x+y, \sigma, r+y)$ is our new partial solution with an increment constraint $c$ and a $T$-invariant $y$, any realizable solution $x+y+z$ obtainable from $p s^{\prime}$ can also be reached from $p s$ by first adding a constraint $c^{\prime}$ for the $T$-invariant $z$ (and later $c, y$ ). If no transition of $z$ can be fired after $\sigma, y+z$ is also not realizable after firing $\sigma$. We may be able to mingle the realization of $z$ with the firing of $\sigma$, but that will be reflected by alternate partial solutions (compared to both, $p s$ and $p s^{\prime}$ ). Therefore, not processing $p s^{\prime}$ will not lose any full solutions.

(4) A similar situation occurs for $p s^{\prime}=(\Gamma \cup\{c\}, x+y, \sigma \tau, r)$ with $\wp(\tau)=y$. There is one problem, though. Since we estimated a token need when choosing $c$ and that estimate may be too low, it is possible that while firing $\tau$ we get closer to enabling some transition $t$ in $r$ without actually reaching that limit where $t$ becomes firable. We thus have to check for such a situation (by counting the minimal number of missing tokens for firing $t$ in the intermediate markings occurring when firing $\sigma$ and $\tau$ ). If $\tau$ does not help in approaching enabledness of some $t$ in $r$, we do not need to process $p s^{\prime}$ any further.

(5) Partial solutions should be memorized if possible to avoid using them as input for CEGAR again if they show up more than once.

\section{The COMPlete AlgORIthm}

The following algorithm integrates the results from the previous sections, using a queue of partial solutions as a job queue that is ordered by ascending size of the possible output, i.e. the firing sequence from an initial marking $m$ to a final marking $m^{\prime}$. 


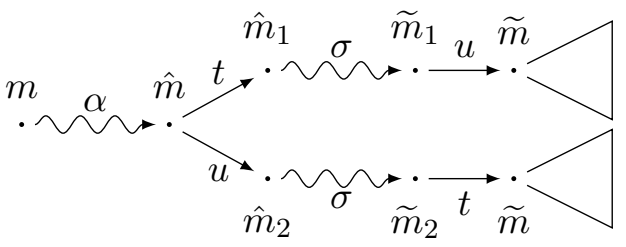

Figure 4: If both sequences $\alpha t \sigma u$ and $\alpha u \sigma t$ can be fired, the subtrees after the nodes with marking $\tilde{m}$ are identical. Only one of the subtrees needs to be evaluated, the other one may be omitted. Snaked lines denote firing sequences

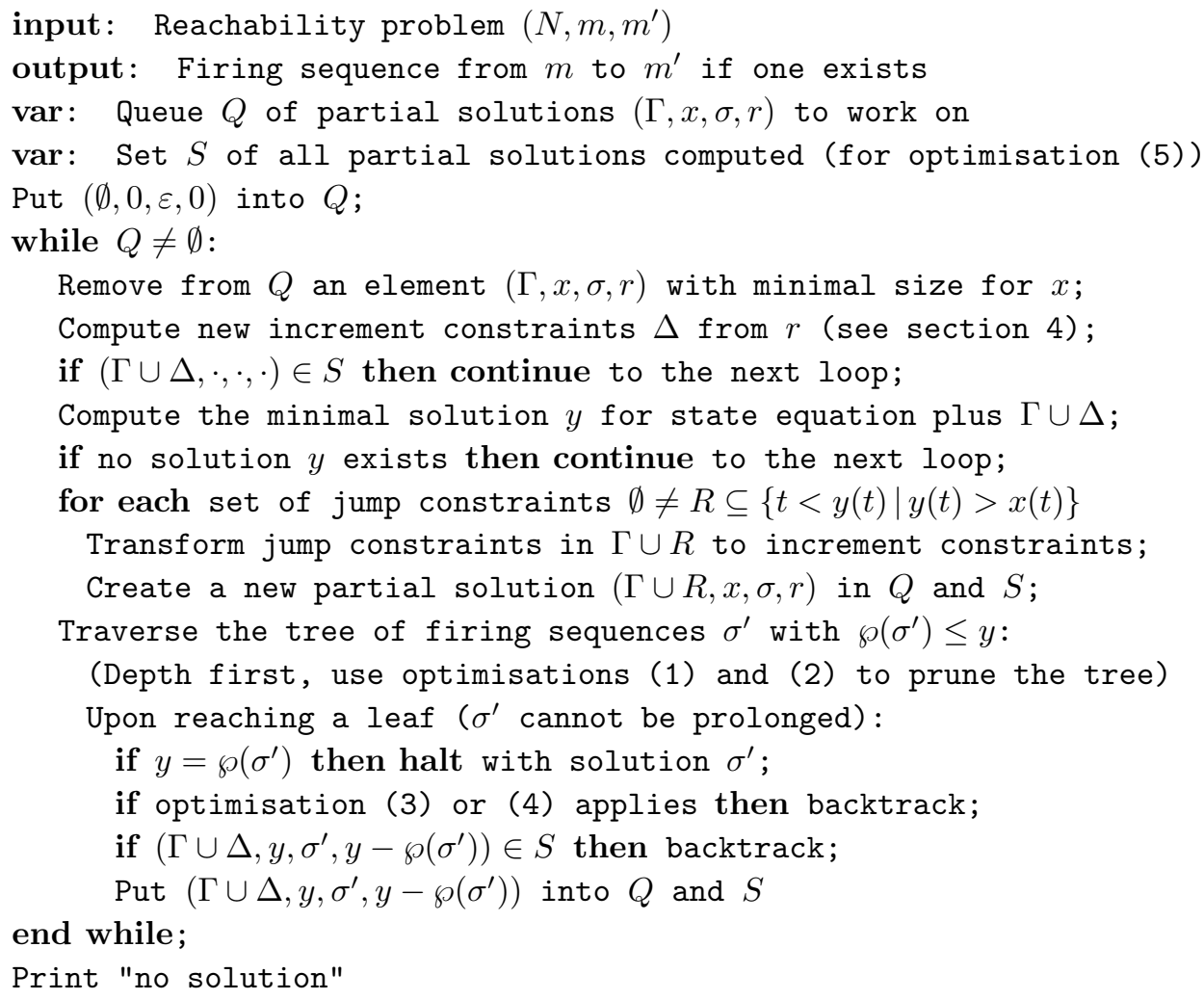

In each while-loop one candidate from the partial solutions queue is processed. First, the constraints $\Delta$ are added according to the non-firable remainder $r$ and a minimal solution vector $y$ is computed. If this is successful, jump constraints are added to the old solution $x$ to obtain other new solutions in a later loop. The minimal solution vector $y$ is then checked for realizable firing sequences. If at some point a firing sequence is incomplete and not extendable, a new partial solution is generated to be able to add more increment constraints later on. The result "no solution" can be obtained by two mechanisms: a partial solution is thrown away if no solution vector $y$ can be computed and the optimisations may prune the trees of firing sequences so much that no new partial solutions are created anymore. There is no guarantee for termination, of course, but in practise this works well.

Note that new jump constraints can lead to an exponential growth in the number of partial solutions, as one such partial solution is created for each subset of transitions where the new solution exceeds the old one. Usually, there are only few different partial solutions, 
i.e. we have just a problem with space limitations but not really with computation time. Thus, we advise to modify the algorithm such that the new partial solutions created from jump constraints are not introduced all at once but one at a time, each time a former one has been processed. If a full solution is found, the unprocessed jump constraints are deleted before they consume space and time, if no full solution exists our experience says that jump constraints are scarce anyway.

\section{Diagnostic Information for UnReachability}

If one of the optimizations 3 or 4 occurs, we know that an increment constraint $c$ added to our system of linear equations did not have the desired effect. This means, we wanted to increase the number of tokens on some set of places but were not able to do so, i.e. a $T$-invariant either was not firable or it had some side effect cancelling the usefulness of the token increase. Such a thing could happen for example if we added a transition $t^{\prime \prime}$ to Fig. 1 that consumes a token from each $s_{2}$ and $s_{3}$ and tried to fire it. The $T$-invariant $u+u^{\prime}$ will produce a token on $s_{2}$, where we need it, but takes away that token from $s_{3}$, cancelling its positive effect. Even if we fire this $T$-invariant more than once, it is of no use.

Compare the partial solutions $p s=(\Gamma, x, \sigma, r)$ and $p s^{\prime}=(\Gamma \cup\{c\}, x+y, \sigma, r+y)$ resp. $p s^{\prime}=(\Gamma \cup\{c\}, x+y, \sigma \tau, r)$ from optimizations 3 and 4. It is obvious that adding the constraint $c$ had not the desired effect, i.e. $c$ failed. We were not able to obtain enough tokens on some set of places $S_{i}$ (that was the reason for introducing $c$ ) to enable some transitions $T_{i}$ (or $X_{i}$, see section 4). If we memorize $S_{i}$ and $T_{i} / X_{i}$ and the number of tokens $n$ missing on $S_{i}$ together with $c$, we can now give a (partial) reason for unreachability. We need at least $n$ more tokens on the set $S_{i}$ to fire one transition in $T_{i}$ or all transitions in $X_{i}$ after the firing sequence $\sigma$. Indeed, $S_{i}$ produces a kind of deadlock: $S_{i} \cup T_{i}$ forms a component of the net $N$ that is strongly connected and the transitions of $T_{i} \cup X_{i}$ cannot fire after $\sigma$ since there are not enough tokens left in $S_{i}$. There may be other transitions though, that could put tokens onto $S_{i}$. If our partial solutions could not be extended with them to obtain a full solution, these transitions are either dead after firing $\sigma$ or they lead to a system of state equation plus constraints that can not be fulfilled. In the latter case, after firing such a transition the final marking becomes unreachable.

Even if we find a reason for unreachability, there may still be other paths that lead to a solution. But if the algorithm terminates altogether (which we are not able to guarantee) and we have not found any solution, we can pick up our failed constraints and present them as diagnostic information. Fig [5 in the next section shows a visualization of such information in a small example Petri net. Hence, our tool cannot only tell the user that a certain marking is unreachable but can also give hints as to where the bottlenecks of the token distribution are that cannot be passed.

\section{Experimental Results}

The algorithm presented here has been implemented in a tool named Sara [19]. We compare Sara to LoLA [20], a low level analyzer searching the (reduced) state space of a Petri net. According to independent reports, e.g. [17, LoLA performs very well on reachability queries and possibly is the fastest tool for standard low level Petri nets. The following tests, realworld examples as well as academic constructions, were run on a $2.6 \mathrm{GHz} \mathrm{PC}$ with $4 \mathrm{~GB}$ 
RAM under Windows XP and Cygwin. While the CPU had four cores, only one was used for the tools. Tests on a similar Linux system lead to comparable but slightly faster results.

- 590 business processes with about 20 up to 300 actions each were tested for "relaxed soundness". Relaxed soundness means that, for each transition $t$ of the net, it is possible to reach the final marking from the initial marking with a path that contains $t$. This problem can be solved using the methods presented above for reachability. The occurrence of $t$ can be asserted by an additional constraint $t>0$ to the IP solver. The processes were transformed into Petri nets and for each action a test was performed to decide if it was possible to execute the action and reach the final state of the process afterwards. Successful tests for all actions/transitions yield relaxed soundness. Sara was able to decide relaxed soundness for all of the 590 nets together (510 were relaxed sound) in 198 seconds, which makes about a third of a second per net. One business process was especially hard and took 12278 calls to lp_solve and 24 seconds before a decision could be made. LoLA was unable to solve 17 of the problems (including the one mentioned above) and took 24 minutes for the remaining 573 .

- Four Petri nets derived in the context of verifying parameterized boolean programs (and published on a web page [8]) were presented to us to decide coverability. Sara needed less than one time slice of the CPU per net and solved all instances correctly. LoLA was not able to find the negative solution to one of the problems due to insufficient memory (here, tests were made with up to $32 \mathrm{~GB}$ RAM), the remaining three problems were immediately solved.

- In 2003, H. Garavel [7] proposed a challenge on the internet to check a Petri net derived from a LOTOS specification for dead (i.e. never firable) transitions. The net consisted of 776 transitions and 485 places, so 776 tests needed to be made. Of the few tools that succeeded, LoLA was the fastest with about 10 minutes, but it was necessary to handle two of the transitions separately with a differently configured version of LoLA. In our setting, seven years later, LoLA needed 41 seconds to obtain the same result. Sara came to the same conclusions in 26 seconds. In most cases the first solution of lp_solve was sufficient, but for some transitions it could take up to 15 calls to lp_solve. Since none of the 776 transitions is dead, Sara also delivered 776 firing sequences to enable the transitions, with an average length of 15 and a longest sequence of 28 transitions. In 2003 the best upper bound for the sequences lengths was assumed to be 35, while LoLA found sequences of widely varying length (the longest having several thousand transition occurrences), though most were shorter than 50 transitions.

- We investigated five nets that represent biochemical reaction chains. The nets stem from the Pathway Logic Assistent [17] where LoLA is integrated for solving reachability problems. For some of the nets (which have several hundred places and transitions), LoLA was incapable of verifying reachability. Sara was able to solve the problems. For three systems, Sara ran less than a second, the remaining two problems could be solved in approximately half an hour. In one of these problem instances the given marking was unreachable.

- Using specifically constructed nets with increasing arc weights (and token numbers) it was possible to outsmart Sara - the execution times rose exponentially with linearly increasing arc weights, the first five times being $0.1,3.3,32,180$, and 699 seconds. LoLA, on the other hand, decided reachability in less than 3 seconds (seemingly constant time) in these cases. 


\begin{tabular}{|c|c|c|c|c|c|c|c|}
\hline Net & Inst. & Sol? & Full & $\neg 1$ & $\neg 2$ & $\neg 3 / 4$ & $\neg 5$ \\
\hline garavel & 776 & 15 & $26 \mathrm{~s}(0.11)$ & $25 \mathrm{~s}(0.11)$ & $26 \mathrm{~s}(0.11)$ & $26 \mathrm{~s}(0.11)$ & $26 \mathrm{~s}(0.11)$ \\
\hline bad-bp & 142 & - & $24 \mathrm{~s}(85)$ & $24 \mathrm{~s}(85)$ & $24 \mathrm{~s}(85)$ & $24 \mathrm{~s}(85)$ & NR \\
\hline good-bp & 144 & 53 & $1.7 \mathrm{~s}(0)$ & $\overline{1.7 \mathrm{~s}(0)}$ & $1.7 \mathrm{~s}(0)$ & $1.7 \mathrm{~s}(0)$ & $1.7 \mathrm{~s}(0)$ \\
\hline test7 & 10 & 175 & $29 \mathrm{~s}(13)$ & $990 \mathrm{~s}(22)$ & NR & $49 \mathrm{~s}(14)$ & $29 \mathrm{~s}(13)$ \\
\hline test8-1 & 1 & 40 & $0.1 \mathrm{~s}(13)$ & $0.35 \mathrm{~s}(22)$ & $49 \mathrm{~s}(13)$ & $0.2 \mathrm{~s}(14)$ & $\overline{0.11 \mathrm{~s}(13)}$ \\
\hline test8-2 & 1 & 76 & $3.3 \mathrm{~s}(21)$ & $24 \mathrm{~s}(51)$ & NR & $11 \mathrm{~s}(34)$ & $3.8 \mathrm{~s}(21)$ \\
\hline test8-3 & 1 & 112 & $32 \mathrm{~s}(27)$ & $390 \mathrm{~s}(80)$ & NR & $175 \mathrm{~s}(71)$ & $33 \mathrm{~s}(27)$ \\
\hline test9 & 1 & - & $\overline{0.4 \mathrm{~s}(53)}$ & $22 \mathrm{~s}(464)$ & NR & NR & $0.9 \mathrm{~s}(65)$ \\
\hline
\end{tabular}

Table 1: Results for shutting down one heuristic. Inst. is the number of problem instances to be solved for the net, Sol? the average solution length or "_" if no solution exists. Columns Full, $\neg 1, \neg 2, \neg 3 / 4$, and $\neg 5$ contain the result with all optimizations, without stubborn sets, without subtree cutting, without partial solution cutting, and without saving intermediate results (numbers are according to Sect. 51). Each entry shows the elapsed time and the number of necessary CEGAR steps (average), or NR if no result could be obtained in less than a day

In addition to these experiments, Sara participated in the Model Checking Contest [6] that was organized within the workshop on Scalable and Usable Model Checking for Petri Nets and Other Models of Concurrency in June 2011 in Newcastle upon Tyne. In this contest, Sara competed in reachability queries on place/transition Petri nets representing a flexible manufacturing system, a KANBAN system, and a biochemical reaction chain. Experiments were done on increasing state spaces that were obtained by adding additional tokens (up to several hundred) on certain places. Sara was able to solve most problem instances in less than a second, the remaining cases were solved within a few seconds. Only two queries led to memory overflow. Sara outperformed all other participating tools including LoLA.

We also checked our heuristics from Sect. 5 with some of the above nets by switching the former off and comparing the results (see Table 1). Our implementation needs both forms of constraints, jump and increment, to guarantee that all solutions of the state equation can be visited. Going through these solutions in a different order, e.g. the total order $\Omega$, is difficult and a comparison was not possible so far.

The nets tested fall in two categories. Garavel's net and the business processes are extensive nets with a low token count and without much concurrency that could be tackled by partial order reduction. The heuristics have no effect here, short runtimes result from finding a good solution to the state equation early on. Only for the hardest of the business processes (bad-bp) memorizing intermediate results to avoid checking the same partial solution over and over made sense - without it we did not get a result at all.

The other category are compact nets. In our test examples a high number of tokens is produced and then must be correctly distributed, before the tokens can be removed again to produce the final marking. With a high level of concurrency in the nets, partial order reduction is extremely useful, the cutting off of already seen subtrees(2) even more than the stubborn set method(1). In the last net (test9), the sought intermediate token distribution is unreachable but the state equation has infinitely many solutions. Only by cutting off infinite parts of the solution tree with the help of optimization 3 and 4 it becomes possible to solve the problem at all. Without them, the number of outstanding CEGAR steps reaches 1000 within less than a minute and continues to increase monotonically. The 


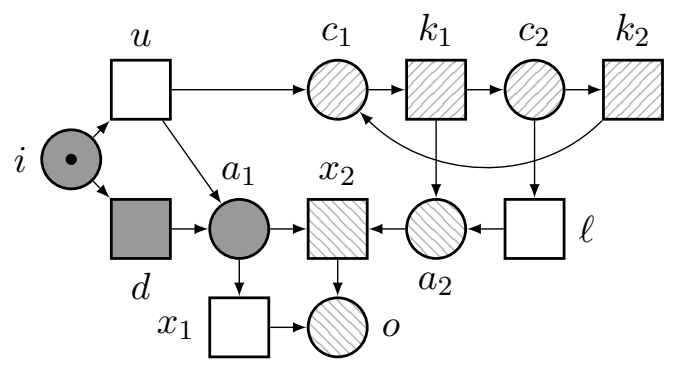

Figure 5: A condensed, flawed business process. One token should flow from the initial place $i$ to the output place $o$ with all other places empty finally. Non-white transitions appear in Sara's solution to the state equation, but only the dark gray one is fireable. Ascending stripes show the area with non-fireable transitions where additional tokens could not be generated

algorithm slows down more and more then as the solutions to the state equation and thus the potential firing sequences become larger.

As mentioned in the previous section, Sara can also provide diagnostic information for unreachable problem instances as long as the state equation has a solution. This feature was tested e.g. with the hardest of the 590 business processes from above, which provides such a negative case for some of its 142 transitions. Using the diagnostic information, we were able to understand the reason for unreachability thus validating the result computed by Sara. Since we cannot present such a large net here, a condensed version with the same important features is shown in Fig. 5 ,

Sara provides a partitioning of the net showing where the relaxed soundness test (for any of the transitions $k_{1}, k_{2}$, or $x_{2}$ ) fails, e.g. it is impossible to fire $x_{2}$ and afterwards reach the final marking with exactly one token on place $o$ (other places being empty). The solution $d+k_{1}+k_{2}+x_{2}$ of the state equation can neither be realized nor extended to a "better" solution. The ascending pattern shows a region of the net (given by Sara) where tokens are needed but cannot be generated without violating the state equation. The descending pattern marks areas that are affected by the former ones, i.e. areas with also non-fireable transitions. The gray transition $d$ is the only fireable transition occurring in the solution. When analyzing the net we can see that the cycle $c_{1}-k_{1}-c_{2}-k_{2}$ indeed constitutes a flaw for a business process: if the cycle gets marked and then emptied later, at least two tokens must flow through $a_{2}$, one of which can never be removed. Using $u$ instead of $d$ is therefore impossible, i.e. $d x_{1}$ is the only firing sequence reaching the final marking.

\section{Towards Parallel Execution}

In essence, Sara solves and evaluates a large number of IP problems corresponding to partial solutions. Different partial solutions are processed mostly independently. In addition, Fig. 3 suggests that our search space is in fact a tree. It is thus natural to consider a parallelization scheme where new threads are opened whenever there is more than one possibility to add a constraint to a partial solution.

The network traffic for opening a new thread is rather low. The Petri net input as well as the problem instance can be loaded by each thread independently and ahead of the actual activation. Then, only the description of a partial solution, especially a set of additional 
constraints, needs to be transmitted. Compared to the network traffic, the internal work involves solving at least one IP problem including subsequent analysis.

Hence, the crucial factor for feasibility of this approach is the branching factor in the search space. With branching factor, we mean the number of new subproblems that are introduced upon the analysis of a partial solution. If the branching factor is one, the subproblems appear sequentially and parallelization does not make sense. Larger values suggest better parallelization results.

We measured the branching factors in several of the examples listed in the previous section. Among those instances that were solved by Sara in less then a second, the maximum branching factor was two while the average branching factor was close to one. In nontrivial examples with practical background (e.g. the hard instances of biochemical reaction chains), the maximal branching factor was two. The average branching factor ranged between 1.25 and 1.99, so there is enough room for feeding many independent threads. Substantial speedup can be expected for processing the large number of IP instances (beyond 160.000). In academic challenges, the maximum branching factor was four, with 2.6 being a typical average value.

We conclude, that the potential for parallel execution is excellent, especially in those problem instances where it is most needed.

\section{Conclusion}

We proposed a promising technique for reachability verification. It is based on the Petri net state equation that naturally provides an overapproximation of the set of reachable states of Petri net. Using the idea of counterexample guided abstraction refinement, we were able to significantly improve the precision of the technique. Our approach has several advantages compared to state space techniques:

(1) It is very efficient, for two reasons. First, we traverse the set of solutions of the state equation rather than the set of all reachable states. That is, our search space is already substantially constrained. Second, we employ the very mature technology of IP solving. We could validate the efficiency in a large number of challenging examples as well as in the independent assessment made in the model checking contest in 2011.

(2) It tends to produce very small (not necessarily minimal) witness paths. This is due to the gradual introduction of period vectors to a minimal solution of the state equation. Unlike explicit state space techniques, short witnesses come without exponentially blowing up the search space. In this regard, the state equation as such resembles a symbolic state representation. Indeed, one solution to the state equation represents up to exponentially many different firing sequences.

(3) It has the tendency to terminate early on unreachable problem instances. In several cases, the initial state equation may already assert unreachability. In contrast, state space techniques (whether explicit or symbolic) cannot benefit from the on-the-fly paradigm if the target state is unreachable. So they would produce all the reachable states modulo the applied state space reduction techniques.

(4) It has a rather pleasant memory consumption. As IP solving is an NP complete problem, polynomial space is sufficient for the core procedure. Only the management of open subproblems may require arbitrary space.

(5) It has an excellent potential for parallelization. Internal executions (IP solving) are nontrivial while network traffic (transmitting a problem description) is rather lightweight. 
(6) It produces some kind of diagnostic information for unreachable problem instances. This potential must be further explored. In particular, usefulness of the diagnostics must be assessed in studies with independent users unaware of the internal mechanisms of Sara.

On the negative side, we need to mention that our approach is incomplete. We are not able to show termination of our procedure and a guaranteed termination may even contradict the EXPSPACE hardness of the reachability problem in general. On the other hand, our experience with Sara so far is very encouraging.

Our approach applies the concept of counterexample guided abstraction refinement in a novel context: the abstraction is not given as a transition system but as a linear-algebraic overapproximation of the reachable states.

The state equation as such has been used earlier for verification purposes, see for instance [5]. In [16], it is used as an initial way of narrowing the state space exploration but not refined according to the CEGAR.

\section{REFERENCES}

[1] 4ti2 team. 4ti2 — A software package for algebraic, geometric and combinatorial problems on linear spaces. http://www.4ti2.de/, 2010.

[2] M. Berkelaar, K. Eikland, and P. Notebaert. lp_solve Reference Guide. http://lpsolve.sourceforge.net/, 2010.

[3] G. Ciardo, R. Marmorstein, and R. Siminiceanu. The saturation algorithm for symbolic state space exploration. Software Tools for Technology Transfer, 8(1):4-25, 2006.

[4] E. Clarke, O. Grumberg, S. Jha, Y. Lu, and H. Veith. Counterexample-guided abstraction refinement. In Proc. $C A V, 2000$.

[5] J. Esparza, S. Melzer, and J. Sifakis. Verification of safety properties using integer programming: Beyond the state equation. Formal Methods in System Design, 16 (2)(2):159-189, 2000.

[6] F. Kordon et al. Report on the model checking contest at Petri nets 2011. To appear in ToPNoC, 2012.

[7] H. Garavel. Efficient Petri Net tool for computing quasi-liveness. http://www.informatik.unihamburg.de/cgi-bin/TGI/pnml/getpost?id=2003/07 /2709, 2003.

[8] G. Geeraerts, J. F. Raskin, and L. Van Begin. Expand, enlarge and check. http://www.ulb.ac.be/di/ssd/ggeeraer/eec/, 2010.

[9] S. R. Kosaraju. Decidability of reachability in vector addition systems. In Proceedings of the 14th Annual ACM STOC, pages 267-281, 1982.

[10] L. M. Kristensen, K. Schmidt, and A. Valmari. Question-guided Stubborn Set Methods for State Properties. Formal Methods in System Design, 29(3):215-251, 2006.

[11] J. L. Lambert. A structure to decide reachability in Petri nets. Theoretical Computer Science, 99:79-104, 1992.

[12] J. Leroux. The General Vector Addition System Reachability Problem by Presburger Inductive Invariants. In Proceedings of the 24th Annual IEEE Symposium on Logic in Computer Science, pages 4-13, Los Alamitos, CA, USA, 2009. IEEE Computer Society.

[13] R. J. Lipton. The Reachability Problem Requires Exponential Space. Research Report, 62, 1976.

[14] E. Mayr. An algorithm for the general Petri net reachability problem. SIAM Journal of Computing, 13(3):441-460, 1984.

[15] K. Schmidt. Stubborn sets for standard properties. In Applications and Theory of Petri Nets 1999: 20th International Conference, ICATPN'99, Williamsburg, Virginia, USA, June 1999. Proceedings, volume 1639 of Lecture Notes in Computer Science, pages 46-65. Springer-Verlag, June 1999.

[16] K. Schmidt. Narrowing Petri net state spaces using the state equation. Fundamenta Informaticae, 47 (3-4):325-335, 2001.

[17] C. Talcott and D. Dill. The pathway logic assistant. In Third International Workshop on Computational Methods in Systems Biology, 2005.

[18] A. Valmari and Henri Hansen. Can stubborn sets be optimal? In Johan Lilius and Wojciech Penczek, editors, Petri Nets, volume 6128 of Lecture Notes in Computer Science, pages 43-62. Springer, 2010. 
[19] H. Wimmel. Sara - Structures for Automated Reachability Analysis. http://servicetechnology.org/tools/download, 2010.

[20] K. Wolf. Generating Petri net state spaces. In ICATPN 200\%, LNCS 4546, pages 29-42. Springer, 2007.

This work is licensed under the Creative Commons Attribution-NoDerivs License. To view a copy of this license, visit http://creativecommons.org/licenses/by-nd/2.0/ or send a letter to Creative Commons, 171 Second St, Suite 300, San Francisco, CA 94105, USA, or Eisenacher Strasse 2, 10777 Berlin, Germany 